\title{
Frecuencia de nematodos gastrointestinales en ovinos de tres distritos de la Región Ancash, Perú
}

\author{
Frequency of gastrointestinal nematodes in sheep from three districts \\ of the Ancash Region, Peru
}

Adhelí Del Carmen Ninamancco C. ${ }^{1}$, Rosa Pinedo V. ${ }^{1}$, Amanda Chávez V. ${ }^{1,2}$

\section{Resumen}

El objetivo del presente estudio fue determinar la frecuencia de nematodos gastrointestinales en ovinos de tres distritos de la Región Ancash, Perú, así como evaluar su asociación con las variables edad ( $\leq 1$ y $>1$ año), sexo y distrito de procedencia (Caraz, Pampas, Yungay). Además, establecer el promedio de carga parasitaria e identificar los géneros y especies presentes. Se colectaron muestras de heces de 540 ovinos durante agosto a setiembre de 2017. Los animales no habían sido tratados con antihelmínticos en los últimos tres meses. Se emplearon las técnicas de flotación con solución de Sheather y McMaster Modificado para exámenes fecales cualitativos y cuantitativos, respectivamente; así como las técnicas de Corticelli y Lai Modificado y la Técnica de Baermann para el cultivo e identificación de larvas de nematodos, respectivamente. La frecuencia de animales positivos a nematodos fue de $79.1 \%$. La prueba de Chi cuadrado no mostró asociación significativa entre la frecuencia de nemátodos y las variables edad, sexo y procedencia. Los promedios geométricos de la carga parasitaria en ovinos positivos a huevos tipo Strongylus (HTS) y Nematodirus sp fueron de 190 y 29 huevos por gramo de heces, respectivamente; considerándose cargas bajas. Los géneros y especies de nematodos más frecuentes fueron Trichostrongylus (80.4\%), Haemonchus contortus (11.4\%) y Oesophagostomum (8.2\%).

Palabras clave: ovejas, nematodos gastrointestinales, gastroenteritis verminosa, Áncash

\footnotetext{
${ }^{1}$ Laboratorio de Microbiología y Parasitología Veterinaria, Facultad de Medicina Veterinaria, Universidad Nacional Mayor de San Marcos, Lima, Perú

${ }^{2}$ E-mail: achavezv@unmsm.edu.pe
}

Recibido: 17 de julio de 2020

Aceptado para publicación: 20 de enero de 2021

Publicado: 24 de abril de 2021 
The aim of this study was to determine the frequency of gastrointestinal nematodes in sheep from three districts of the Ancash Region, Peru, as well as to evaluate their association with the variables age ( $\leq 1$ and $>1$ year), sex and district of origin (Caraz, Pampas, Yungay). In addition, establish the average parasite load and identify the genera and species present. Faecal samples were collected from 540 sheep during August to September 2017. The animals had not been treated with anthelmintics in the last three months. Sheather's and Modified McMaster's flotation techniques were used for qualitative and quantitative faecal examinations, respectively; as well as the Corticelli and Lai Modified techniques and the Baermann technique for the culture and identification of nematode larvae, respectively. The frequency of nematode positive animals was $79.1 \%$. The Chi square test did not show a significant association between the frequency of nematodes and the variables age, sex and origin. The geometric averages of the parasite load in sheep positive to Strongylus (HTS) and Nematodirus sp eggs were 190 and 29 eggs per gram of faeces, respectively, and considered as low loads. The most frequent nematodes genuses and species were Trichostrongylus sp (80.4\%), Haemonchus contortus (11.4\%) and Oesophagostomum sp (8.2\%).

Key words: sheep, gastrointestinal nematodes, verminous gastroenteritis, Ancash

\section{INTRODUCCIÓN}

En el Perú, la crianza de ovinos esta difundida en las tres regiones naturales, Costa, Sierra y Selva, debido a su capacidad de adaptación a diferentes climas, siendo de mayor importancia en la zona altoandina entre $\operatorname{los} 3000$ y $4200 \mathrm{msnm}$ (Aliaga, 2012). Esta práctica genera ingresos y empleo a más de un millón de familias campesinas, quienes la integran con otros tipos de crianzas como la de vacunos y camélidos (Díaz, 2007; Aliaga, 2012; Ramírez, 2012).

La crianza de los ovinos en el país es considerada como un sistema con inversión cero o nula y de bajos rendimientos, que solo resulta rentable en explotaciones pecuarias mega-dimensionadas. Esta situación se vio influida por la disminución del precio internacional de la lana gruesa y media, falta de dinamismo de la industria para adaptarse a los cambios de mercado, falta de innovación en sistemas productivos, y depredación de las pasturas naturales y mal manejo de los re- cursos hídricos (Vivanco, 2012; Ramírez, 2012).

La nematodiasis gastrointestinal (gastroenteritis verminosa) es una parasitosis de gran repercusión en la rentabilidad de las explotaciones pecuarias, siendo una de las principales causas de pérdidas económicas en la producción ovina (Lacasta et al., 2008). Afecta el crecimiento de los animales, pérdida de peso, baja digestibilidad y anemia, conllevando a una baja eficiencia productiva (Radostis et al., 1999; Bikila et al., 2013; Muluneh et al., 2014). Asimismo, el uso inapropiado de antiparasitarios ocasiona fallas en la eficacia de los antihelmínticos y conlleva al desarrollo de resistencia (Soulsby, 1987; Kassai, 2002; Steffan et al., 2012).

El parasitismo gastrointestinal se encuentra distribuido a nivel mundial afectando la economía de los ganaderos y de las familias campesinas. Así, estudios realizados en Brasil reportaron prevalencias de nemátodos gastrointestinales del 50.3\% (Da Silva et al., 2010 ) y en Venezuela de $43.6 \%$ de infeccio- 
nes por estróngilos (Quijada et al., 2006). En Colombia, Bolivia, y México se han reportado prevalencias de $97.7 \%$ (Ensuncho-Hoyos, 2014), $90 \%$ (Rodríguez, 2004) y $77.6 \%$ (Rojas et al., 2007), respectivamente. Estudios en el país sobre gastroenteritis verminosa en ovinos indican prevalencias de 35 a $83 \%$ en la Región Tacna (Condori et al., 2017) y 75\% en la SAIS Túpac Amaru Región, Junín (Cabello, 2007).

El objetivo del presente estudio fue determinar la frecuencia de nematodos gastrointestinales presentes en ovinos de tres distritos de la región de Ancash; así como, evaluar su asociación con las variables edad, sexo y procedencia. Además, establecer el promedio de carga parasitaria e identificar los géneros y/o especies de nematodos presentes.

\section{Materiales y Métodos}

\section{Lugar de Estudio}

El estudio se desarrolló entre agosto y setiembre de 2017 en los distritos de Yungay (2458 msnm), Caraz (2285 msnm) y Pampas (3180 $\mathrm{msnm})$, pertenecientes a las provincias de Yungay, Huaylas y Pallasca, respectivamente, departamento de Ancash, Perú. La temperatura máxima/mínima y las precipitaciones esporádicas fueron de $24 / 4{ }^{\circ} \mathrm{C}$ y $8 \mathrm{~mm}$ en Caraz, $25 / 8.4{ }^{\circ} \mathrm{C}$ y $14 \mathrm{~mm}$ en Pampas y $23 / 10^{\circ} \mathrm{C}$ con $10 \mathrm{~mm}$ en Yungay (SENAMHI, 2017). Los distritos fueron seleccionados por las facilidades y preocupación de los municipios de establecer planes de control contra el parasitismo gastrointestinal.

\section{Población y Muestra}

La población de ovinos en el departamento de Ancash era de 24653 animales según el IV Censo Nacional Agropecuario (INEI, 2012). Para determinar el número de ovejas a ser muestreadas, se utilizó la fórmula de poblaciones finitas (Daniel, 2007). Se consideró una prevalencia esperada de $75.29 \%$ (Condori et al., 2017), con un nivel de confianza del $95 \%$ y un error del $5 \%$, obteniéndose una cantidad mínima a muestrear de 286 animales.

Se empleó la fórmula de estratificación (Pérez, 2000) para determinar el número de muestras por comunidad, utilizando la población de ovinos en Yungay (7727), Caraz (3497) y Pampas (13 429) (INEI, 2012), correspondiendo 90,40 y 156 animales por distrito, respectivamente. Sin embargo, con el apoyo voluntario de los pastores residentes de la zona de estudio y el apoyo de los municipios, se pudo recolectar 130, 120 y 290 muestras procedentes de Yungay, Caraz y Pampas, respectivamente, dando un total de 540 ovinos.

Se muestrearon de manera aleatoria animales de ambos sexos y de crianza extensiva pertenecientes a diferentes caseríos. Como criterio de inclusión, los ovinos no debieron recibir tratamiento antihelmíntico en los tres meses previos al estudio y como criterio de exclusión no se muestrearon hembras en los últimos meses de gestación, ni animales menores de seis meses.

Las muestras fecales (10 g) se tomaron directamente del recto y fueron almacenadas en recipientes térmicos con geles refrigerantes para su trasladado al Laboratorio de Parasitología de la Facultad de Medicina Veterinaria de la Universidad Nacional Mayor de San Marcos, Lima, para su procesamiento, dentro de los cinco días de su colección.

\section{Análisis Coproparasitológico}

Se utilizó la técnica de flotación de Sheather para determinar, de manera cualitativa, la presencia de huevos de nematodos. Asimismo, para cuantificar la carga parasitaria o la cantidad de huevos de nematodos por gramo de heces (hpg) se empleó el método de McMaster Modificado (Rojas, 2004; Vignau et al., 2005; Benavides, 2013). 
Para la identificación de las especies de nematodos se realizó un coprocultivo con las muestras fecales que presentaron huevos tipo Strongylus, obteniéndose las larvas de tercer estadio mediante los métodos de Corticelli y Lai Modificado; Baermann (Niec, 1968, Benavides. 2013). La identificación se basó en claves internacionales en función a características larvarias como presencia o ausencia de vaina de la segunda muda, aspecto de la cavidad oral o esófago, número de células intestinales, forma y tamaño según la medición del largo total de la larva y el largo de la cola de la cubierta (Niec, 1968; Vignau et al., 2005; Zajac y Conboy, 2012) a través de la captura digital y procesamiento de imágenes utilizando el programa Leica Application Suite v. 4.0.

\section{Análisis Estadístico}

Se calculó la frecuencia total de nematodos y la media geométrica de los hpgsegún las variables edad ( $\leq 1$ año, $>1$ año), sexo y procedencia (distritos); con sus respectivos intervalos de confianza al 95\%. Se realizó la prueba de Chi cuadrado para evaluar la asociación $(p<0.05)$ entre la frecuencia de nematodos y las tres variables (Daniel, 2007). Asimismo, se halló la frecuencia de cada tipo de huevo de nematodo con sus respectivos intervalos de confianza al $95 \%$ y se evaluó su asociación con las tres variables mediante la prueba de Chi cuadrado $(\mathrm{p}<0.05)$.

La relación entre la carga parasitaria con las variables sexo y edad fue determinada mediante la prueba no paramétrica de Mann Whitney para la variable procedencia por la prueba de Kruskal Wallis (Daniel, 2007). Además, se determinó la frecuencia de géneros y/o especies de larvas infectivas (L3) según los distritos de procedencia. Todos los análisis se realizaron empleando el programa Stata/IC 15.0 (Stata Corp, College Station, TX).
Resultados

E1 79.1\% de los ovinos evaluados en los tres distritos de Áncash resultaron positivos a una o más especies de nematodos. La prueba de Chi cuadrado no encontró diferencia significativa entre las variables evaluadas (edad, sexo y procedencia) con la frecuencia de nematodos (Cuadro 1). Las frecuencias de huevos de nematodos tipo Strongylus y Nematodirus spp fueron de 79.1 y $25.1 \%$, respectivamente. Solo se encontró asociación $(\mathrm{p}=0.009)$ entre la presencia de huevos de Nematodirus spp y la variable procedencia (Cuadro 2).

La media geométrica de las cargas parasitarias de ovinos positivos a huevos tipo Strongylus (HTS) y Nematodirus spp fue de 190 y $29 \mathrm{hpg}$, respectivamente. Se halló asociación significativa $(\mathrm{p}=0.016)$ entre la variable edad y la carga parasitaria de huevos tipo Strongylus, así como entre la variable procedencia con la carga de huevos tipo Strongylus $(\mathrm{p}<0.001)$ y con la carga de huevos de Nematodirus $\operatorname{spp}(\mathrm{p}=0.022)$ (Cuadro 3).

En el cultivo de larvas de se encontró $80.4,11.4$ y $8.2 \%$ de larvas infectivas (L3) correspondientes a Trichostrongylus sp, Haemonchus contortus y Oesophagostomum sp, respectivamente (Figura 1). La frecuencia por distrito se presenta en el Cuadro 4.

\section{Discusión}

Las alteraciones generadas por nematodos conllevan a una disminución de la producción en los animales afectados; siendo considerada como una de las enfermedades que genera mayores pérdidas económicas en la producción de rumiantes menores (Rojas, 2004; Lacasta et al., 2008). En el presente estudio, se halló una alta frecuencia 
Cuadro 1. Frecuencia de nemátodos en ovinos, según edad, sexo y procedencia de tres distritos de Áncash, Perú (2017)

\begin{tabular}{lccccc}
\hline Variables & $\mathrm{n}(\%)$ & Positivos & $\begin{array}{c}\text { Frecuencia } \\
(\%)\end{array}$ & IC (95\%) & $\mathrm{p}^{1}$ \\
\hline $\begin{array}{l}\text { Edad (años) } \\
\quad 1\end{array}$ & $147(27.2)$ & 123 & 83.6 & $75.7-87.3$ & 0.263 \\
$\quad>1$ & $393(72.7)$ & 304 & 77.3 & $74.8-79.9$ & 0.108 \\
Sexo & & & & \\
$\quad$ Hembra & $286(52.3)$ & 219 & 76.6 & $71.2-81.4$ & 0.079 \\
$\quad$ Macho & $254(47.1)$ & 208 & 81.9 & $76.6-86.4$ & \\
Procedencia & & & & & \\
$\quad$ Caraz & $120(22.2)$ & 89 & 74.2 & $65.4-81.7$ & \\
$\quad$ Pampas & $290(53.1)$ & 231 & 79.7 & $74.6-84.1$ & 0.270 \\
$\quad$ Yungay & $130(24.1)$ & 107 & 82.3 & $74.7-88.5$ & \\
\hline Total & 540 & 427 & 79.1 & $75.4-82.3$ & \\
\hline
\end{tabular}

${ }^{1}$ Prueba de Chi cuadrado

Cuadro 2. Frecuencia de tipos de huevos de nemátodos en ovinos $(n=540)$ de tres distritos del Áncash, Perú (2017)

\begin{tabular}{lcccccc}
\hline \multirow{2}{*}{ Variables } & \multicolumn{3}{c}{ Huevos tipo Strongylus (HTS) } & \multicolumn{3}{c}{ Nematodirus $\mathrm{sp}$} \\
\cline { 2 - 7 } & $\%$ & IC 95\% & $\mathrm{p}^{1}$ & $\%$ & IC 95\% & $\mathrm{p}^{1}$ \\
\hline Edad (años) & & & & & & \\
$\quad \leq 1$ & 83.6 & $75.7-87.3$ & 0.108 & 32.6 & $25.4-39.3$ & 0.263 \\
$\quad>1$ & 77.3 & $74.8-79.9$ & & 27.7 & $26.4-28.9$ & \\
Sexo & & & & & & \\
$\quad$ Hembra & 76.6 & $71.2-81.4$ & 0.079 & 27.6 & $22.5-33.2$ & 0.244 \\
Macho & 81.9 & $76.6-86.4$ & & 30.7 & $25.1-36.8$ & \\
Procedencia & & & & & & \\
Caraz & 74.2 & $65.4-81.7$ & 0.270 & 23.3 & $14.5-33.3$ & 0.009 \\
$\quad$ Pampas & 79.6 & $74.6-84.1$ & & 28.3 & $23.2-34.0$ & \\
$\quad$ Yungay & 82.0 & $74.7-88.5$ & & 19.2 & $14.8-29.6$ & \\
\hline Total & 79.1 & $75.4-82.3$ & & 25.1 & $21.3-28.9$ & \\
\hline
\end{tabular}

${ }^{1}$ Prueba de Chi cuadrado 
Cuadro 3. Media geométrica de la carga parasitaria de ovinos positivos a huevos tipo Strongylus (HTS) y Nematodirus sp, de tres distritos del Áncash, Perú (2017)

\begin{tabular}{|c|c|c|c|c|c|c|}
\hline \multirow{2}{*}{ Variables } & \multicolumn{3}{|c|}{ Huevos tipo Strongylus (HTS) } & \multicolumn{3}{|c|}{ Nematodirus sp } \\
\hline & Media & IC 95\% & $\mathrm{p}$ & Media & IC $95 \%$ & $\mathrm{p}$ \\
\hline \multicolumn{7}{|l|}{ Edad (años) } \\
\hline$\leq 1$ & 221 & $178-274$ & \multirow{2}{*}{0.016} & 42 & $37-47$ & \multirow{2}{*}{0.120} \\
\hline$>1$ & 177 & $154-204$ & & 35 & $31-35$ & \\
\hline \multicolumn{7}{|l|}{ Sexo } \\
\hline Hembra & 196 & $167-231$ & \multirow{2}{*}{0.611} & 37 & $33-40$ & \multirow{2}{*}{0.429} \\
\hline Macho & 181 & $152-216$ & & 38 & $35-42$ & \\
\hline \multicolumn{7}{|l|}{ Procedencia } \\
\hline Caraz & 95 & $76-120$ & \multirow{3}{*}{$<0.001$} & 23 & $15-32$ & \multirow{3}{*}{0.022} \\
\hline Pampas & 244 & $212-282$ & & 52 & $48-56$ & \\
\hline Yungay & 192 & $169-214$ & & 28 & $26-31$ & \\
\hline Total & 190 & $169-214$ & & 29 & $25-33$ & \\
\hline
\end{tabular}

Cuadro 4. Porcentaje de distribución de los géneros y/o especie de larvas infectivas (L3) en muestras de heces de ovinos de tres distritos del Áncash, Perú, con altas cargas de huevos tipo Strongylus

\begin{tabular}{ccccc}
\hline \multirow{2}{*}{$\begin{array}{c}\text { Especie de larva infectiva } \\
\text { (L3) }\end{array}$} & \multicolumn{3}{c}{ Procedencia (\%) } & Total \\
\cline { 2 - 4 } & Caraz & Pampas & Yungay & $(\%)$ \\
\hline Haemonchus contortus & 14 & 28 & 14 & 11.4 \\
Oesophagostomum $\mathrm{sp}$ & 7 & 30 & 8 & 8.2 \\
Trichostrongylus $\mathrm{sp}$ & 79 & 42 & 78 & 80.4 \\
\hline
\end{tabular}

(79.1\%) de nemátodos gastrointestinales en ovinos criados de forma extensiva en tres distritos de Ancash, resultado concordante a los reportados en otros países de América (Rodríguez et al., 2004; Rojas et al., 2007; Ensuncho-Hoyos, 2014) y del país (Cabello, 2007, Condori et al., 2017).
El estudio de Ames (1967) en la provincia ancashina de Recuay reportó una prevalencia de $96.26 \%$ en ovinos mediante el recuento diferencial de parásitos post mortem. Estudios adicionales en la zona no han sido reportados según conocimiento de los autores. Si bien los resultados de dicho estudio no 


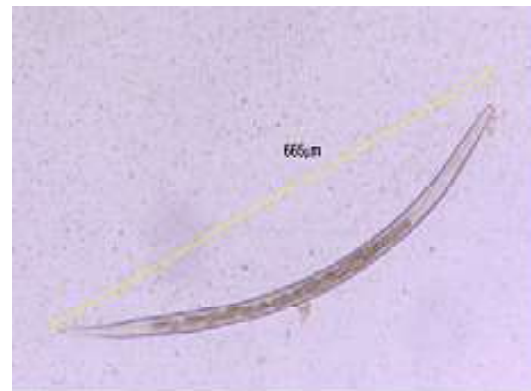

(A)
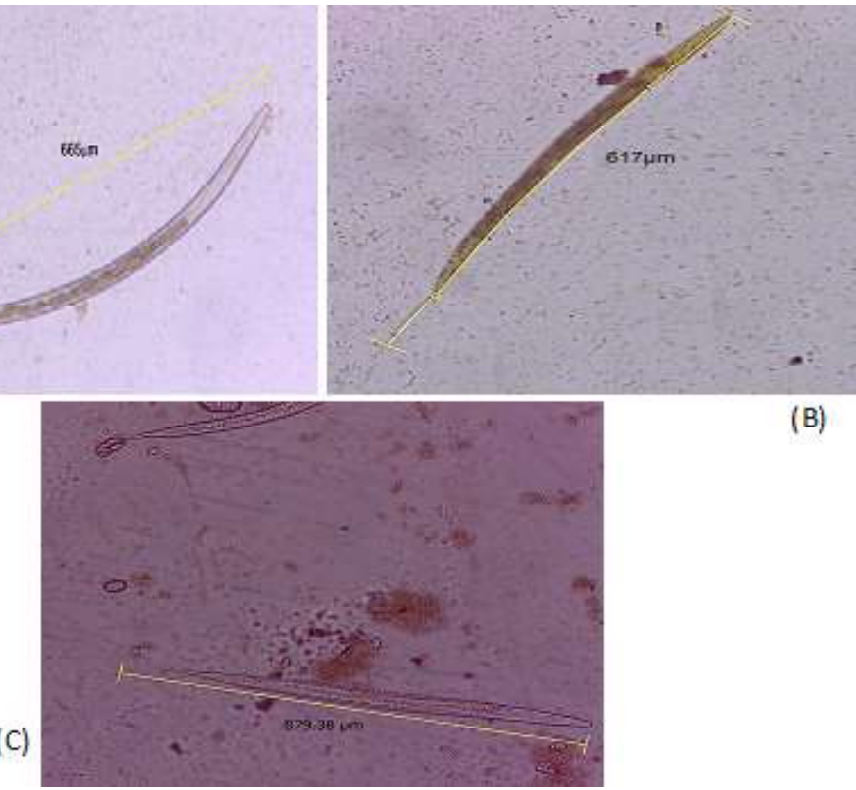

(B)

Figura 1. (A) Larva L3 infectiva de Trichostrongylus sp. Región posterior con vaina corta y achatada; (B) Larva L3 infectiva de Haemonchus contortus. Región posterior, vaina mediana y termina en prolongación filamentosa; (C) Larva L3 infectiva de Oesophagostomum sp. Región posterior, vaina muy larga y filamentosa

pueden ser contrastados con los del presente estudio, dadas las metodologías empleadas para determinar la prevalencia, los valores obtenidos indicarían que la presencia de los parásitos gastrointestinales en ovinos de Ancash continúa siendo elevada.

Diversos estudios reportan que la infección por nemátodos en ovinos estaría influenciada por múltiples factores relacionados al hospedero, parásito y medio ambiente, tales como la edad, tipo de manejo, potencial biótico y diferencias climáticas, entre otros (Rojas, 2004; Roeber et al., 2013; Taylor et al., 2016). En el presente estudio no se encontró una asociación significativa entre la frecuencia de nematodiasis con las variables edad, sexo y procedencia; no obstante, se reconoce que animales menores de un año son más susceptibles a la parasitosis gastrointestinal debido a su sistema inmune inmaduro (Radostis, 1999; Taylor et al., 2016), sucediendo de igual manera en hembras adul- tas gestantes por la depresión inmune periparto (Cordero del Campillo y RojoVázquez, 1999; Da Silva et al., 2010). En este estudio no se hallaron estas diferencias, probablemente, porque no se trabajó con ovinos menores de seis meses ni con hembras gestantes, debido al rechazo de los criadores para muestrear estos animales por temor a causarles daño en la toma de muestra.

La ausencia de asociación significativa entre la frecuencia de parásitos gastrointestinales con el distrito de procedencia se debió, probablemente, a la similitud en el manejo y tipo de crianza extensiva llevado a cabo por los criadores de la zona, quienes pastorean en forma conjunta, tanto animales jóvenes como adultos, contribuyendo a la transmisión en los jóvenes libres de infecciones (Taylor et al., 2016). Factores adicionales para las altas cargas parasitarias encontradas se debería a que el ovino corta el pasto cerca del suelo posibilitando una mayor 
ingesta de estadios larvarios infectivos (Kanyari et al., 2009) y a las condiciones de malnutrición debido a la mala calidad de pasturas (Dos Santos, 2008; Pala, 2011).

La mayor frecuencia de huevos tipo Strongylus (79.1\%) que Nematodirus spp (25.1\%) podría deberse a las condiciones climáticas de la zona, la hipobiosis y la capacidad biótica relacionado al factor parásito (Taylor et al., 2016). La temperatura ambiental de la zona $\left(18-24{ }^{\circ} \mathrm{C}\right)$ con precipitaciones pluviales esporádicas, que fueron incrementando debido a que el muestreo se dio durante la transición de época seca a lluviosa (SENAMHI, 2017), favorece la actividad metabólica de los nemátodos con huevos tipo Strongylus (HTS), generando el desarrollo de las forma infectiva (L3), debido a que este proceso requiere de temperaturas en promedio mayores a $15{ }^{\circ} \mathrm{C}$ (Rojas, 2004, Ghalsasi et al., 2015). Estas larvas, luego de ser ingeridas por los rumiantes inician su fase actividad parásita ya sea en el abomaso (Haemonchus contortus), intestino delgado (Trichostrongylus sp) o grueso (Oesophagostomum $\mathrm{sp}$ ).

Asimismo, las hembras de los nematodos encontrados presentan un alto potencial biótico. Así, Trichostrongylus sp con 100200 huevo/día, Oesophagostomum sp con 3000 huevos/día y Haemonchus contortus con 5000-10000 huevos por día (Ueno y Gonçalves, 1998), mientras que Nematodirus sp presenta un menor potencial biótico (50100 huevos por día) y necesitan un mayor tiempo para el desarrollo de las larvas infectivas dentro del huevo. Además, algunas especies de Nematodirus sp requieren cambios bruscos de temperaturas (noches frías seguidos de días cálidos) para facilitar la eclosión de los huevos, por lo que su número generalmente suele ser mayor en la época seca (Rojas, 2004).

Las condiciones medioambientales y el potencial biótico indicado explicarían la baja carga parasitaria encontrada (Ueno y Gonçalves, 1998; Maichomo et al., 2004), habiéndose encontrado promedios geométricos de 190 y $29 \mathrm{hpg}$ para el tipo Strongylus y Nematodirus sp, respectivamente. Estos resultados son concordantes con los hallados por Lupaca (2017) y Condori et al., (2017) en Tacna (754 hpg y 133 hpg tipo Strongylus, respectivamente) en comparación con cargas de 178 hpg y 133 hpg de Nematodirus spp, respectivamente. Las diferencias significativas observadas entre la media geométrica de la carga parasitaria, respecto a la edad y procedencia, no representarían diferencias biológicas de importancia, dado que las cargas halladas fueron bajas. De otra parte, la mayor carga parasitaria en los ovinos de Pampas podría deberse al mayor número de animales muestreados en ese distrito en comparación los otros dos distritos.

La alta frecuencia de larvas L3 de Trichostrongylus spp (80.4\%), seguido de una menor frecuencia de Haemonchus contortus (11.4\%) y Oesophagostomum spp (8.2\%), fueron relativamente similares a los resultados obtenidos por Ames (1967) en el distrito de Recuay, Áncash (Trichostrongylus sp: $51.4 \%$ y Oesophagostomum sp: $27.3 \%$ ), aunque no reportaron $H$. contortus, debido a que ese distrito presenta un clima muy frío $\left(5{ }^{\circ} \mathrm{C}\right)$ y seco, no ideal para el desarrollo de este nematodo (O'Connor et al., 2006). Del mismo modo, la baja frecuencia de Oesophagostomum sp en el presente estudio podría explicarse por la época de muestreo, ya que agosto es un mes muy frío, con temperaturas promedio de $5{ }^{\circ} \mathrm{C}$ y en el mes de septiembre recién se inicia el incremento de la temperatura en la zona del estudio, entendiéndose que este nematodo requiere temperaturas mayores $\left(20-22{ }^{\circ} \mathrm{C}\right)$ (O'Connor et al., 2006).

\section{Conclusiones}

- La frecuencia de nemátodos gastrointestinales en ovinos de tres distritos del departamento de Áncash fue de 79.1\% con un intervalo de confianza al 95\% de 
$75.4-82.3 \%$, sin encontrar asociación significativa con las variables procedencia, sexo y edad.

- Se hallaron huevos tipo Strongylus y Nematodirus sp con frecuencias de $79.1 \%$ (IC $95 \%: 75.4-82.3 \%$ ) y $25.1 \%$ (IC al 95\%: 21.3-28.9\%), respectivamente, y con medias geométricas de cargas parasitarias bajas de $190 \mathrm{hpg}$ (169-214 hpg) y $29 \mathrm{hpg}$ (25-33 hpg), respectivamente.

- Se identificaron larvas infectantes L3 de Haemonchus contortus (11.4\%), Oesophagostomum sp (8.2\%). y Trichostrongylus sp (80.4\%).

\section{Limeratura Citada}

1. Aliaga JL. 2012. Producción de ovinos. Lima: Univ. Nacional Agraria La Molina. $299 \mathrm{p}$.

2. Ames LG 1967. Encuesta de helmintos gastrointestinales en ovinos de Recuay (departamento de Ancash). Tesis de Médico Veterinario. Lima: Univ. Nacional Mayor de San Marcos. 60 p.

3. Benavides OE. 2013. Técnicas para el diagnóstico de endoparásitos de importancia veterinaria. Bogotá: Univ. de La Salle. 94 p.

4. Bikila E, Yeshitla A, Worku T, Teka F, Benti D. 2013. Epidemiology of gastrointestinal parasites of small ruminants in Gechi district, southwest Ethiopia. Adv Biol Res 7: 169-174. doi: 10.5829/idosi.abr.2013.7.5.74176

5. Cabello IC. 2007. Frecuencia de helmintiasis gastrointestinal y coccidiosis en heces de ovino de la SAIS Túpac Amaru. Tesis de Médico Veterinario. Lima: Univ. Nacional Mayor de San Marcos. 94 p.

6. Condori STJ, Ramos MLA, Galindo SWM, Coaquira RJC. 2017. Prevalencia de parásitos gastrointestinales en ovinos de la provincia Jorge Basadre Tacna. UNJBG. [Internet]. Disponible en: http://ugpc.unjbg.edu.pe/pdfpro-
yecto/0112201705530TeodoraCondori2016\%20 Prevalencia\% $20 \mathrm{de} \% 20$ parásitos $\% 20$ gastrointestinales\%20en.pdf

7. Cordero del Campillo M, RojoVázquez FA. 1999. Parasitología veterinaria. Madrid: McGraw-Hill. 987 p.

8. Da Silva M, De Souza E, Bonelli E, Medeiros M; Da Silva G. 2010. Parasitas gastrintestinais de ovinos criados na regiao de Rondonópolis. Rev Biodiv 9(7). [Internet]. Disponible en: http://periodicoscientificos.ufmt.br/ojs/ index.php/biodiversidade/article/view/ 102/93

9. Daniel W. 2007. Bioestadística base para el análisis de las ciencias de la salud. $4^{\mathrm{a}}$ ed. México: Limusa. $924 \mathrm{p}$.

10. Díaz RI. 2007. Sector ovinos con perspectivas al 2015. MINAGRI. [Internet]. Disponible en: http://www.produccionanimal.com.ar/produccion_ovina/ produccion_ovina/106-diaz_ovinos_peru.pdf

11. Dos Santos NM. 2007. Sobrevivencia y migración de larvas de nemátodos gastrointestinales de ovinos. Tesis de Ingeniero Zootecnista. Florianópolis: Univ. Federal De Santa Catarina. 30 p.

12. Ensuncho-Hoyos C, Castellano CA, Maza AL, Bustamante YM, Vergara GO. 2014. Prevalencia y grado de infección de nemátodos gastrointestinales en ovinos de pelo en pastoreo de cuatro municipios de Córdoba, Colombia. Rev Cient-Fac Cien V 24: 414-420.

13. Ghalsasi PM, Ghalsasi PP, Bandgar RS, Nimbkar C. 2015. Indications of hypobiosis of Haemonchus contortus in goats of deccan Plateau in Maharashtra. Ind J Small Ruminants 21: 354-355. doi: 10.5958/0973-9718.2015.00083.5

14. [INEI] Instituto Nacional de Estadística e Informática. 2012. IV Censo nacional agropecuario. Informe Definitivo. [Internet], Disponible en: http:// proyectos.inei.gob.pe/web/DocumentosPublicos/ResultadosFinalesIVCENAGRO.pdf 
15. Kanyari PWN, Kagira JM, Mhoma RJ. 2009. Prevalence and intensity of endoparasites in small ruminats kept by framers in Kisumu Municipality, Kenya. Livestock Res Rural Dev 21(11). [Internet]. Disponible: http://www.lrrd.org//rrd21/11/kany21202.html

16. Kassai T. 2002. Helmintología veterinaria. Zaragoza: Acribia. 258 p.

17. Lacasta D, Ferrer LM, Ramos JJ, Calvete C, Uriarte J, Ruiz M, Ortega M. 2008. Resistencia a los antiparasitarios de uso común en ganaderías ovinas de Aragón. FEADER 198(8). [Internet]. Disponible: https://citarea.cita-aragon.es/ citarea/bitstream/10532/886/1/1053297_2.pdf

18. Lupaca JA. 2017. Prevalencia de parásitos gastrointestinales en ovinos de la raza Hampshire Down (Ovis aries) del distrito de Sama, Tacna 2016. Tesis de Médico Veterinario y Zootecnista. Tacna: Univ. Nacional Jorge Basadre Grohmann. $89 \mathrm{p}$.

19. Maichomo MW, Kagira JM, Walker T. 2004. The point prevalence of gastrointestinal parasites in calves, sheep and goats in Magadi division, south western Kenya. J Vet Res 71: 257.261. doi: 10.4102/ojvr.v71i4.229

20. Muluneh J, Bogale B, Chanie M. 2014. Major gastrointestinal nematodes of small ruminants in Dembia district, Northwest Ethiopia. Eur J Appl Sci 6: 30-36. doi: 10.5829/idosi.ejas.2014.6.2 .8652

21. Niec R. 1968. Cultivo e identificación de larvas infectantes de nematodes gastrointestinales del bovino y ovino. Manual Técnico 3. [Internet]. Disponible en: http://helminto.inta.gob.ar/Niec/ Cultivo\%20e\%20Identificaci\%C3\%B3n\%20de \%20Larvas\%-20Infectantes\%20de.pdf

22. O'Connor LJ, Walkden-Brown SW, Kahn LP. 2006. Ecology of the freeliving stages of major trichostrongylide parasites of sheep. Vet Parasitol 142: 115. doi: 10.1016/j.vetpar.2006.08.035
23. Pala LP. 2011. Determinación de los tiempos de reinfestación de las cargas parasitarias (parásitos pulmonares, gastrointestinales y hepáticos), en ovinos de la estación de altura Moyocancha ubicada a $3600 \mathrm{msnm}$ perteneciente a la Espoch. Tesis de Ingeniero Zootecnista. Rio Bamba: Escuela Superior Politécnica de Chimborazo. $111 \mathrm{p}$.

24. Pérez C. Técnicas de muestreo estadístico. México: Alfa Omega. 603 p.

25. Quijada J, García F, Vivas I, Simoes D, Rondón Z. 2006. Prevalencia de infecciones por estróngilos digestivos en un rebaño ovino del estado de Aragua en la época de lluvia. Rev Cient-Fac Cien V Luz 16: 341-346.

26. Radostis O, Gay C, Blood D, Hinchcliff K. 1999. Tratado de las enfermedades del ganado bovino, ovino, porcino, caprino y equino. $9^{\mathrm{a}}$ ed. Madrid: McGraw-Hill. 1037 p.

27. Ramírez E. 2012. Manual de crianza de ganado ovino. Lima: Globalmark. $48 \mathrm{p}$.

28. Roeber F, Jex A, Gasser R. 2013. Impact of gastrointestinal parasitic nematodes of sheep, and the role advanced molecular tools for exploring epidemiology and drug resistance an Australian perspective. Parasite Vector 6: 153. doi: 10.1186/1756-3305-6-153

29. Rodríguez IJL. 2004. Determinación de la carga parasitaria e identificación de nemátodes gastrointestinales en ovinos de pelo en San Julián «Cuatro Cañadas» provincia Ñuflo de Chávez del departamento de Santa Cruz. Tesis de Médico Veterinario y Zootecnista. Santa Cruz, Bolivia: Univ. Autónoma Gabriel Rene Moreno. 49 p.

30. Rojas M. 2004. Nosoparasitosis de los rumiantes domésticos peruanos. $2^{\mathrm{a}}$ ed. Lima: Martegraf. $154 \mathrm{p}$.

31. Rojas HS, Gutiérrez SI, Olivares PJ, Valencia AMT. 2007. Prevalencia de nemátodos gastrointestinales en ovinos en pastoreo en la parte alta del MPIO De Cuetzalá del Progreso, Guerrero México. REDVET 8(9). [INTERNET]. 
Disponible en: https://www.researchgate.net/publication/26475878_Prevalencia de nematodos gastrointestinales en_ovinos_en_pastoreo_en_la parte_alta_del_MPIO_De_Cuetzala_del_Progreso_GuerreroMexico

32. [SENAMHI] Servicio Nacional de Meteorología e Hidrología. 2017. Datos hidrometeorológicos a nivel nacional. [Internet]. Disponible en: https:// www.senamhi.gob.pe/?\&p=estaciones

33. Soulsby EJL. 1987. Parasitología y enfermedades parasitarias en los animales domésticos. $7^{\mathrm{a}}$ ed. México: Interamericana. $810 \mathrm{p}$.

34. Steffan PE, Fiel CA, Ferreyra DA. 2012. Endoparásitos más frecuentes de los rumiantes en sistemas pastoriles de producción. Buenos Aires: Grupo Reencuentro. $118 \mathrm{p}$.
35. Taylor M.A, Coop R.L, Wall R.L. 2016. Veterinary parasitology. $3^{\circ}$ ed. Oxford: Blackwell. $600 \mathrm{p}$.

36. Ueno H, Gonçalves PC. 1998. Manual para diagnóstico das helmintoses de ruminantes. $4^{\circ}$ ed. Tokio: Japan International Cooperation Agency. $144 \mathrm{p}$.

37. Vivanco H. 2012. Reconversión y mejora genética de la ganadería ovina y caprina en el Perú. Agrobanco. [Internet]. Disponible en: https://www.agrobanco.com.pe/pdf_cpc/ReconversionMejoraGenetica.pdf.

38. Vignau M, Venturini L, Romero J, Eiras D, Basso W. 2005. Parasitología práctica y modelos de enfermedades parasitarias en los animales domésticos. La Plata: Univ. Nacional La Plata. 194 p.

39. Zajac A, Conboy G 2012. Veterinary clinical parasitology. $8^{\text {th }}$ ed. Iowa: Wiley. $327 \mathrm{p}$. 